\title{
Recommendations and Clinical Guidance for Children with Metabolic-associated Liver Disease during the COVID-19 Pandemic
}

\author{
Yong-Hai Zhou" ${ }^{\oplus} \oplus$, Rafael S. Rios $\# 2 \oplus$, Kenneth I. Zheng $\# 2 \oplus$ \\ and Ming-Hua Zheng ${ }^{* 2,3,4}$
}

${ }^{1}$ Department of Pediatrics, The Second Affiliated Hospital and Yuying Children's Hospital of Wenzhou Medical University, Wenzhou, Zhejiang, China; ${ }^{2}$ MAFLD Research Center, Department of Hepatology, The First Affiliated Hospital of Wenzhou Medical University, Wenzhou, Zhejiang, China; ${ }^{3}$ Institute of Hepatology, Wenzhou Medical University, Wenzhou, Zhejiang, China; ${ }^{4}$ Key Laboratory of Diagnosis and Treatment for the Development of Chronic Liver Disease in Zhejiang

Province, Wenzhou, Zhejiang, China

Citation of this article: Zhou YH, Rios RS, Zheng KI, Zheng $\mathrm{MH}$. Recommendations and clinical guidance for children with metabolic-associated liver disease during the COVID-19 pandemic. J Clin Transl Hepatol 2021;000(000):000000. doi: $10.14218 /$ JCTH.2020.00137

The outbreak of coronavirus disease-2019 (COVID-19), a new disease caused by the severe acute respiratory syndrome coronavirus 2 (SARS-CoV-2) in December 2019 affected most countries in the world within just a few months. By March 11, 2020, the Director-General at the World Health Organization (WHO) declared that COVID-19 can be considered a pandemic, and as of January 11, 2021, there were over 88.8 million cases of COVID-19 worldwide and 1.9 million deaths reported by WHO. In order to prevent the further spread of this epidemic outbreak, measures such as social distancing, stay-at-home orders, and school closure were executed in many countries. However, these measures also greatly reduced the opportunities for physical activity among children. ${ }^{1}$ Based on past experience, there are indications that an increased rate of weight gain occurs during summer school vacation for overweight children. ${ }^{2}$ Therefore, it is reasonable to speculate that under the influence of the COVID-19 epidemic, long-term school closures may significantly increase the risk of weight gain in obese children. For children with metabolic-associated fatty liver disease (MAFLD), 3,4 formerly known as nonalcoholic fatty liver disease, weight reduction through lifestyle changes (caloric restriction and physical activity) is considered as the first step in reversing disease progression, which makes managing MAFLD in children quite difficult during the ongoing COVID-19 pandemic. In addition, several studies found a significant correlation between a higher risk of severe illness from COVID-19 and metabolic disorders (e.g. diabetes, obesity, MAFLD). ${ }^{5-7}$

Keywords: COVID-19; MAFLD; Children.

Abbreviations: COVID-19, coronavirus disease-2019; DHA, docosahexaenoic acid; MAFLD, metabolic-associated fatty liver disease; SARS-CoV-2, severe acute respiratory syndrome coronavirus 2; WHO, World Health Organization. Received: 28 November 2020; Revised: 13 January 2021; Accepted: 15 January 2021

\#These authors contributed equally to this study.

*Correspondence to: Ming-Hua Zheng, MAFLD Research Center, Department of Hepatology, the First Affiliated Hospital of Wenzhou Medical University, No. 2 Fuxue Lane, Wenzhou, Zhejiang 325000, China. Tel: +86-577-55579622, Fax: +86-577-55578522, E-mail: zhengmh@wmu.edu.cn

\section{Challenges and risks}

\section{Reduced physical activity}

Due to the closure of schools, public swimming pools, football stadiums and other sports venues, outlets for physical activities in children have decreased significantly, especially for urban children, due to the small venues available in the living area. While this helps to avoid cross-infection in the community, staying at home frequently equates to less physical activity.

\section{Sedentary and increased electronic screen exposure time}

Due to the decrease in social activities, many children tend to spend more time watching television shows and playing video games, which also leads to less physical activity.

\section{Unhealthy diet}

Due to the impact of the epidemic, travel restrictions in many places have reduced shopping behavior, while limited transportation has caused a reduction in the distribution of supplies; collectively, these have led to an increased intake of unhealthy food (frozen meat, fast-food delivery service, etc.), and reduced intake of the healthier fresh vegetables and fruits. More exposure time to electronic screens and less social activities outside the house also leads to increased consumption of snacks, such as potato chips, and many children will experience a higher calorie intake in their diet.

\section{Response measures and recommendations}

\section{To the clinicians}

MAFLD is often described as a disease without symptoms and has a time scale of decades. Therefore, clinical visits for pediatric MAFLD patients are not recommended at this time. Whether COVID-19 may affect the progression of MAFLD is unknown. This is because the evolution of COVID-19 in the 
near future is uncertain and specialized medicine to treat SARS-CoV-2 infection is still in the early stage of development. MAFLD-cirrhosis children may require a clinical visit every 3-6 months, although it is recommended to avoid going to the hospital if possible.

There are presently no approved pharmacologic drugs for the treatment of MAFLD, but numerous drugs for fatty liver have entered phase II or phase III clinical trials. Although studies have shown that vitamin $\mathrm{E}$ and docosahexaenoic acid (commonly known as DHA) have a certain role in improving liver steatosis in children, they are known to not be effective in reducing alanine aminotransferase. Probiotics might prove to be an effective treatment strategy. ${ }^{8}$ In addition, for children with MAFLD, the safety of drugs should be considered, especially the risk of side effects affecting growth and development.

\section{To the children with MAFLD and their guardians}

For this population, the following are important guidelines:

a) Choose safe and ventilated places for physical activities and avoid crowded or indoor places, such as swimming pools. For children with restrictions to sports venues, it is recommended to choose physical activities, such as skipping, that do not require a specific location. Once outside of the home, it is recommended to wear masks on the road and wash hands frequently.

b) Guardians should spend more time with children and engage in physical activities to reduce the exposure time to electronic products, such as television programs and video games.

c) Guardians should provide children with a healthy diet, especially of fresh vegetables and fruits, and reduce the consumption of fatty food and high-calorie snacks, such as potato chips and carbonated beverages.

d) Since current studies suggest that abnormal liver function and obesity seem to predict poor clinical evolution in patients with COVID-19, guardians are recommended to keep note of the child's weight during the course of social distancing and be aware of the negative consequences of weight gain toward MAFLD.

\section{Summary}

It is estimated that the prevalence of MAFLD is $7.6 \%$ in nonobese children and $34.2 \%$ in obese children. ${ }^{7}$ Because more and more adult MAFLD patients need liver transplantation due to liver cirrhosis, it is necessary to intervene resolutely in children with MAFLD in the early stages of life in order to reverse or delay the progress of the disease. This necessity is further emphasized when taking into consideration that even milder forms of liver damage or dysfunction have shown a relation to worse outcomes in COVID-19 patients. ${ }^{9}$
As we have previously reported, obesity increases the risk for severe COVID-19 illness, ${ }^{6}$ especially among MAFLD patients, for whom the risk is more than 6 -fold. ${ }^{10}$ Therefore, prevention of SARS-CoV-2 infection in conjunction with weight control in pediatric MAFLD patients (who are usually already obese) during the COVID-19 epidemic is very important, especially when considering that the SARS-Cov-2 virus may coexist with humans for a long time to come and that the current global spread has not yet been effectively contained.

\section{Funding}

This work was supported by grants from the National Natural Science Foundation of China (82070588), High Level Creative Talents from Department of Public Health in Zhejiang Province (S2032102600032) and Project of New Century 551 Talent Nurturing in Wenzhou.

\section{Conflict of interest}

The authors have no conflict of interests related to this publication.

\section{References}

[1] Rundle AG, Park Y, Herbstman JB, Kinsey EW, Wang YC. COVID-19-related school closings and risk of weight gain among children. Obesity (Silver Spring) 2020;28:1008-1009. doi:10.1002/oby.22813.

[2] Franckle R, Adler R, Davison K. Accelerated weight gain among children during summer versus school year and related racial/ethnic disparities: a systematic review. Prev Chronic Dis 2014;11:E101. doi:10.5888/pcd11.130355.

[3] Eslam M, Newsome PN, Sarin SK, Anstee QM, Targher G, Romero-Gomez $\mathrm{M}$, et al. A new definition for metabolic dysfunction-associated fatty liver disease: An international expert consensus statement. J Hepatol 2020;73:202-209. doi:10.1016/j.jhep.2020.03.039.

[4] Zheng KI, Fan JG, Shi JP, Wong VW, Eslam M, George J, et al. From NAFLD to MAFLD: a "redefining" moment for fatty liver disease. Chin Med J (Engl) 2020;133:2271-2273. doi:10.1097/CM9.0000000000000981.

[5] Targher G, Mantovani A, Wang XB, Yan HD, Sun QF, Pan KH, et al. Patients with diabetes are at higher risk for severe illness from COVID-19. Diabetes Metab 2020;46:335-337. doi:10.1016/j.diabet.2020.05.001.

[6] Gao F, Zheng KI, Wang XB, Sun QF, Pan KH, Wang TY, et al. Obesity is a risk factor for greater COVID-19 severity. Diabetes Care 2020;43:e72e74. doi: $10.2337 / \mathrm{dc} 20-0682$

[7] Zhou YJ, Zheng KI, Wang XB, Yan HD, Sun QF, Pan KH, et al. Younger patients with MAFLD are at increased risk of severe COVID-19 illness: A multicenter preliminary analysis. J Hepatol 2020;73:719-721. doi:10.1016/j. jhep.2020.04.027.

[8] Nobili V, Alisi A, Valenti L, Miele L, Feldstein AE, Alkhouri N. NAFLD in children: new genes, new diagnostic modalities and new drugs. Nat Rev Gasdren: new genes, new diagnostic modalities and new drugs. Nat Rev Gas-

[9] Targher G, Mantovani A, Byrne CD, Wang XB, Yan HD, Sun QF, et al. Risk of severe illness from COVID-19 in patients with metabolic dysfunction-associated fatty liver disease and increased fibrosis scores. Gut 2020;69:15451547. doi:10.1136/gutjnl-2020-321611.

[10] Zheng KI, Gao F, Wang XB, Sun QF, Pan KH, Wang TY, et al. Letter to the Editor: Obesity as a risk factor for greater severity of COVID-19 in patients with metabolic associated fatty liver disease. Metabolism 2020;108:154244. doi:10.1016/j.metabol.2020.154244. 